\title{
HILBERT-TYPE INEQUALITIES INVOLVING DIFFERENTIAL OPERATORS, THE BEST CONSTANTS, AND APPLICATIONS
}

\author{
VAndanjaV Adiyasuren, Tserendorj Batbold And Mario Krnić
}

Abstract. Motivated by some recent results, in this article we derive several Hilbert-type inequalities with a differential operator, regarding a general homogeneous kernel. Moreover, we show that the constants appearing on the right-hand sides of these inequalities are the best possible. The general results are then applied to some particular examples of homogeneous kernels and compared with previously known from the literature.

Mathematics subject classification (2010): Primary 26D10, 26D15, Secondary 33B15.

Keywords and phrases: Hilbert-type inequality, Hardy inequality, differential operator, homogeneous function, Gamma function.

\section{REFERENCES}

[1] M. Abramowitz, I. A. STEgun, Handbook of mathematical functions with formulas, graphs, and mathematical tables, National Bureau of Standards, Applied Math. Series 55, 4th printing, Washington 1965.

[2] L. E. AzAR, Two new forms of Hilbert-type integral inequality, Math. Inequal. Appl., 17, 3 (2014), 937-946.

[3] A. ČIŽMEŠIJA, M. KRnIĆ, J. PEČARIĆ, General Hilbert's inequality with non-conjugate parameters, Math. Inequal. Appl. 11 (2008), 237-269.

[4] R. L. Graham, D. E. Knuth, D. Patashnik, Concrete mathematics - A Foundation for Computer Science, Addison-Wesley Publishing Company, 1989.

[5] G. H. HARDY, Note on some points in the integral calculus, LXIV, Messenger Math. 57 (1928), 12-16.

[6] G. H. Hardy, J. E. Littlewood, G. Pólya, Inequalities, $2^{\text {nd }}$ edition, Cambridge University Press, Cambridge, 1967.

[7] M. KRniĆ, J. PeČAriĆ, General Hilbert's and Hardy's inequalities, Math. Inequal. Appl. 8 (2005), $29-52$.

[8] M. Krnić, G. Mingzhe, J. PeČarić, G. Xuemei, On the best constant in Hilbert's inequality, Math. Inequal. Appl. 8 (2005), 317-329.

[9] A. Kufner, L. Maligranda, L. E. Persson, The Hardy inequality - About its history and some related results, Vydavatelský servis, Pilsen, 2007.

[10] I. Perić, P. VuKović, Hardy-Hilbert's inequalities with a general homogeneous kernel, Math. Inequal. Appl. 12 (2009), 525-536. 